\title{
Assembly and Disassembly of Bare Chips using On-Substrate Linear Micro Vibromotor Arrays
}

\author{
Kazuhiro Saitou \\ Soungjin J. Wou \\ Dung-An Wang \\ Design Laboratory \\ Department of Mechanical Engineering and Applied Mechanics \\ University of Michigan, Ann Arbor, MI 48109-2125, USA \\ \{kazu,jasonwou,wangd $\} @$ engin.umich.edu
}

\begin{abstract}
A new method for on-substrate fine positioning of micro/meso-scale discrete components is proposed, where component positions are finely adjusted using micro linear sliders and fixtures on the substrate. Each micro linear slider is actuated by vibratory impacts exerted by two pairs of micro cantilever impacters. These micro cantilever impacters are selectively resonated by shaking the entire substrate with a piezoelectric vibrator, realizing forward and backward slider motion to facilitate assembly and disassembly of a micro component on the substrate. An array of the prototype externally-resonated linear micro vibromotors is fabricated using the MCNC MUMPS service. These prototypes are tested for forward and backward motion via external vibration applied by a piezoelectric stack vibrator. The linear micro vibromotor array is to be integrated in a on-chip micro assembly device where the assembly of micro/meso-scale discrete components (bare chips) on a common substrate is done by the combination of vibratory palletization for gross positioning, and linear micro vibromotor for fine positioning.
\end{abstract}

\section{Introduction}

The increasing demand for the size reduction in integrated micro systems has directed the higher density interconnection among micro electrical components. This yielded the concept of bare chip interconnection, where unpackaged VLSI chips are interconnected directly on a common substrate. Compare to the conventional printed circuit board assembly, bare chip interconnection has advantages in not only the dramatic size reduction, but also in achieving high functional densities, lowest possible propagation delays and improved reliability and system performance.

Currently, interconnections of bare chips on a common substrate is achieved either by wire bonding, tape automated assembly (TAB), or flip-chip bonding technologies [7]. As a result, the system reliability, which to a large extent depends upon the integrity of each of the interconnecting solder joints in the system, is reduced. In addition, the solder joints are virtually permanent once they are established, which renders the overall system less modular. For example, the entire system must be discarded even in the event of a subsystem failure. Decreased modularity also causes an entire system to be replaced even in the case of subsystem upgrade. These technical problems would have high environmental impacts with the increasing number of production of the micro systems utilizing the bare chip interconnection technologies. This motivates the development of a high-precision assembly/disassembly method for mesoscale components which require high-density electrical interconnection. Although the size of the chips currently used for bare chip interconnection are typically $5-10 \mathrm{~mm}$, the advent of such assembly/disassembly method would stimulate further disintegration of subsystem components to improve the overall system modularity, which in turn would reduce the sizes of the chips to be assembled.

Despite such motivation, no practical assembly/disassembly methods of micro/meso-scale components suitable for automation has been developed so far. This is mainly due to the surface adhesion forces causing sticking among components and handling devices [5], which makes simple miniaturization of the conventional pick-and-place robotic assembly extremely difficult. Figure 1 illustrates pick-and-place assembly of a micro-scale component using a micro gripper. Surface adhesion forces such as electrostatic, van der Waals, and surface tension forces cause the component to stick to the gripper during the approach (Figure 1 (b)) and the release (Figure 1 (d)) phases. Mechanical shock can be applied to the gripper to drop the stuck component (Figure $1(\mathrm{e})$ ), with the price of inaccurate positioning of the released component (Figure 1 (f)). 

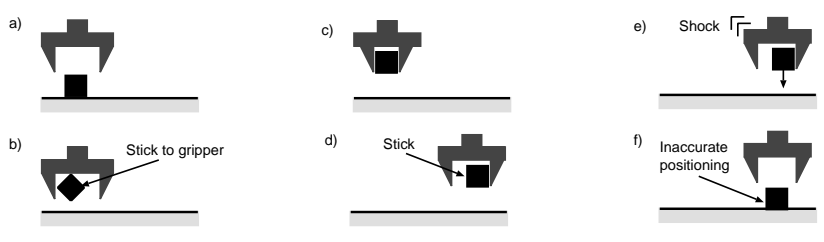

Figure 1. Typical pick-and-place assembly in micro scale (modified from [5]).
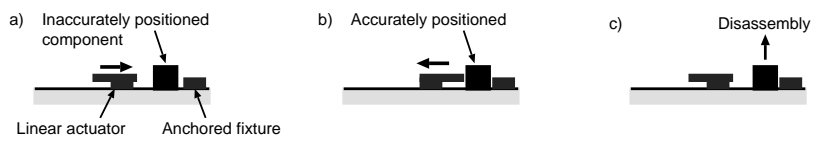

Figure 2. Precise component positioning and release with a on-substrate linear actuator and a fixture.

One way to overcome this problem is to design a device on the substrate that facilitates component positioning so that gross positioning is done in the conventional pickand-place fashion ${ }^{1}$, whereas fine positioning is done by the on-substrate positioning device. This concept is illustrated in Figure 2, where a on-substrate linear actuator pushes a inaccurately positioned micro component (e.g., as a result of the "shock release" shown in Figure 1 (e) and (f)) against a fixture anchored to the substrate (Figure 2 (a)), achieving precise positioning of the component (Figure 2 (b)). The linear actuator also should be able to re-open to release the positioned component to facilitate the potential needs for disassembly (Figure 2 (c)).

This paper describes a design of such a micro linear actuator for fine positioning of a micro/meso-scale discrete component on a substrate. The design is based on a linear micro vibromotor reported by Daneman et al. [4], where a micro linear slider is actuated by vibratory impacts exerted by micro cantilever impacters. Dissimilar to their design, however, these micro cantilever impacters are selectively resonated by shaking the entire substrate with a piezoelectric vibrator, requiring no need for built-in driving mechanisms such as electrostatic comb actuators. This selective resonance of the micro cantilever impacters via an external vibration energy field [15] provides with a very simple means of controlling forward and backward motion of the micro linear slider, facilitating assembly and disassembly of a micro component on a substrate. An array of prototype linear micro vibromotor are fabricated using the three-layer polysilicon surface micro machining process provided by

\footnotetext{
${ }^{1}$ or with other processes which are more effective for gross positioning - see Section 4 for an example.
}

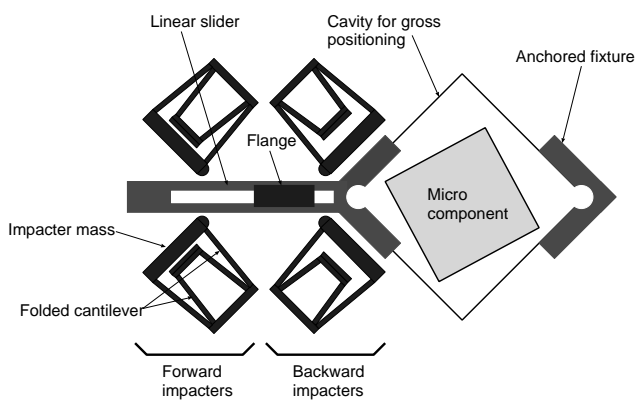

Figure 3. A schematic top view of the externally-resonated linear micro vibromotor for on-substrate precise positioning.

the MCNC MUMPS service. These prototypes are tested for forward and backward motion via external vibration applied by a piezoelectric stack vibrator.

\section{Design}

\subsection{Operational principle}

Our design of the externally-resonated linear micro vibromotor for micro assembly is based on a linear micro vibromotor reported by Daneman et al. [4], where a micro linear slider is actuated by vibratory impacts exerted by micro cantilever impacters. Dissimilar to their design, however, these micro cantilever impacters are selectively resonated by external piezoelectric vibration, requiring no need for built-in driving mechanisms such as electrostatic comb actuators.

As illustrates in Figure 3, it consists of a linear slider located between two pairs of folded cantilever impacters anchored on the substrate which can exert forward and backward vibratory impacts to the sides of the slider, depending on which pair of impacters is resonated by external vibration. Figure 4 illustrates the three-step operation of the linear micro vibromotor. First, the substrate is shaken with a piezoelectric vibrator at the frequency $f_{1}$. This external vibration resonates only the forward impacters, causing the linear slider to move right (Figure 4 (a)). This motion causes the slider to push a micro component against an anchored fixture, achieving precise positioning (Figure 4 (b)). Next, the substrate is shaken at the frequency $f_{2}$. This external vibration resonates only the backward impacters and moves the slider to the left (Figure 4 (c)), releasing the positioned component.

This selective resonance of the micro cantilever impacters via an external vibration energy field [15] provides with very simple means of controlling forward and back- 

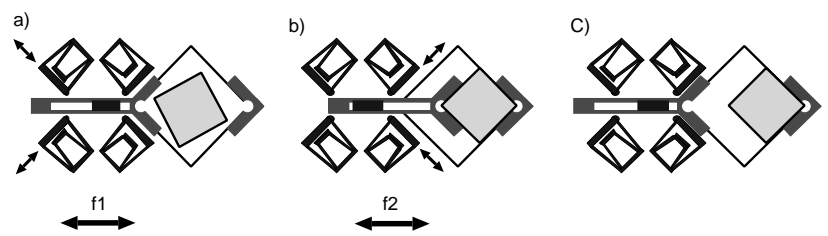

\section{Figure 4. Three-step operation of the externally-resonated linear micro vibromotor.}

ward motion of the micro linear slider, without explicit routing to direct energy to each of the impacters. This property of the selective resonance would be particularly useful in the situation where a number of linear micro vibromotors are implemented in a two-dimensional array in order to position multiple micro components simultaneously. By designing the forward and backward impacters to have different resonance frequencies, each linear micro vibromotor in the array can be operated independently by the external piezoelectric vibrations driven by the sum of the signals with appropriate resonance frequencies [15].

In Figure 4, note that the direction of the external vibration is not parallel to the direction of impacters' oscillation (i.e., the direction of impact). Therefore, it is the component of the external vibration parallel to the direction of impact that causes the resonance in the micro impacters. Another component of external vibration causes the impacters to deform perpendicular to the direction of impact, which is undesirable for efficient operation of the linear micro vibromotor. The micro cantilever impacters, therefore, should have high stiffness in the direction perpendicular to the direction of impact, while keeping the relatively low stiffness in the direction of impact. To achieve this goal, the double- $\mathrm{V}$ beam suspension design [10] is employed in the micro cantilever impacters, which realizes higher transversal stiffness than the conventional folded parallel beam design without affecting the lateral stiffness [10].

\subsection{Modeling}

Equations of motions of a lumped parameter model of the impacter-slider system illustrated in Figure 3 is derived in order to obtain an optimal design which maximizes translation speed of the linear slider at desired external input frequencies. Let $(\zeta, \gamma)$ be the coordinate system for the slider position, with $\zeta$ being the direction of slider motion, and $(x, y)$ be the coordinate system for the impacter position with $x$ being the direction of impact, rotated from $\zeta-\gamma$ axes by the impact angle $\theta$. An impacter can be modeled as a simple mass-spring-damper system with an external force input $f_{e x t}(t)$ :

$$
m \ddot{x}+b \dot{x}+k x=f_{\text {ext }}(t)
$$

where $m, b$, and $k$ are the mass, viscous damping coefficient, and spring constant of a impacter, respectively. Assuming Coutette air flow between the substrate and the impacter mass, and small lateral displacement of the folded beams, these parameters are expressed as $[2,10]$ :

$$
\begin{aligned}
m & =\rho A t+2 \rho h w l \\
b & =\mu \frac{A}{d} \\
k & =E h\left(\frac{w}{l}\right)^{3} \cos \phi\left(1+\frac{\cos \phi}{2}\right)
\end{aligned}
$$

where $\rho$ is the mass density of the impacter material (polysilicon); $A$ and $t$ are the planer area (including the area of the joining member of two folded beams) and thickness of the impacter mass, respectively; $h, w$, and $l$ are the height, width, and total length of the two segments of a Vbeam, respectively; $\mu$ is the viscosity of the air; $d$ is the vertical gap between the substrate and the impacter mass; $E$ is Young's modulus of the beam material (polysilicon); and $\phi$ is the half of the angle between the two segments of a V-beam. Assuming the substrate is shaken with the external vibration $\zeta=Z_{0} \cos (\omega t)$ in $\zeta$ direction, the inertial force $f_{\text {ext }}(t)$ exerted to a impacter is:

$$
f_{\text {ext }}(t)=m \omega^{2} Z_{0} \cos \theta \cos (\omega t)
$$

Similarly, the equation of motion of the linear slider is given as:

$$
M \ddot{\zeta}=F(t)
$$

where $M$ is the mass of the slider and $F$ is a net force exerted to the slider:

$F(t)=\left\{\begin{array}{lr}0 \quad \text { if } \dot{\zeta}=0 \text { and }\left|-B \dot{\zeta}+F_{\text {ext }}(t)\right|<F_{s} \\ -B \dot{\zeta}+F_{\text {ext }}(t)-F_{d}(\dot{\zeta} /|\dot{\zeta}|) \quad \text { otherwise }\end{array}\right.$

where $B$ is the viscous damping coefficient of the slider; $F_{\text {ext }}(t)=M \omega^{2} Z_{0} \cos (\omega t)$ is the inertial force exerted to the slider; $F_{s}$ and $F_{d}$ are static and dynamic frictional forces, respectively. The parameters $M$ and $B$ are given similarly to Equations 2 and 3.

An oblique impact of the impacter tips to the slider side wall is modeled as an impact with restitution in $\gamma$ direction, and an impact with instantaneous momentum transfer in $\zeta$ direction [8]. Let $c$ be the distance between the impacter tip and the slider side wall measured in $x$ direction. If $x<c$, there is no impact. At $x=c$, the impacter tip contacts the slider sidewall. In in $\gamma$ direction, the following boundary condition models the energy dissipation of the impacter at an impact:

$$
V^{+}=V^{-}+2 \frac{m}{M}(1+e) v^{-} \cos \theta
$$


Table 1. The physical constant values used in the simulation

\begin{tabular}{|c|c|}
\hline Parameter & Value [unit] \\
\hline \hline$\rho$ & $2.33\left[\mathrm{~g} / \mathrm{cm}^{3}\right]$ \\
\hline$\mu$ & $1.79 \times 10^{-5}[\mathrm{~Pa} \cdot \mathrm{s}]$ \\
\hline$E$ & $169[\mathrm{GPa}]$ \\
\hline$F_{s}$ & $20[\mu \mathrm{N}]$ \\
\hline$F_{d}$ & $5[\mu \mathrm{N}]$ \\
\hline$e$ & 0.5 \\
\hline
\end{tabular}

where $V^{-}$and $V^{+}$are slider velocities in $x$ direction right before and right after the impact.

The equations of motion defined as Equations 1 through 8 are numerically integrated with the forth-order Runge Kutta method to evaluate the performance of the externallyresonated linear micro vibromotor. The values of the $\phi$ and $\theta$ used in the numerical simulation are $15^{\circ}$ and $45^{\circ}$, respectively. The values of $d, h$, and $t$ are constrained by the MUMPs process provided by MCNC used for device fabrication discussed in Section 3. They are set to be $0.75 \mu \mathrm{m}$, $2.0 \mu \mathrm{m}$, and $3.5 \mu \mathrm{m}$, respectively. The physical constant values used in the simulation are shown in Table 1 . The values of $F_{s}$ and $F_{d}$ account for not only the friction between the substrate and the slider but also the slop between the slider and its guide, and are estimated based on [4] since the slider size and its fabrication process are virtually identical.

Figure 5 shows results of numerical integration of the above equations of motion 1 through 8 in the time period from $t_{0}=0.0[\mathrm{msec}]$ to $t_{f}=3.0[\mathrm{msec}]$ with two external input frequencies: (a) $\nu=\omega / 2 \pi=5.2[\mathrm{kHz}]$ and (b) $\nu=\omega / 2 \pi=6.2[\mathrm{kHz}]$. For each input frequency, the top figure shows the time plot of the slider position, and the bottom figure shows the time plot of the impacter position. The parameter values common to both figures are $A=$ $1.5 \times 10^{4}\left[\mu m^{2}\right], w=4.0[\mu \mathrm{m}], l=600[\mu \mathrm{m}], c=3.0[\mu \mathrm{m}]$, and the slider area is $8.0 \times 10^{4}[\mu \mathrm{m}]$. These values give the impacter natural frequency $\nu_{n}=\omega_{n} / 2 \pi=5.2[\mathrm{kHz}]$, where $\omega_{n}=\sqrt{k / m}$. The initial condition $\left(x\left(t_{0}\right), \dot{x}\left(t_{0}\right)\right)$ is $(0.0,0.0)$ in both cases. Note that the slider moves approximately four times faster when driven with $6.2 \mathrm{kHz}$ (Figure 5 (b)) than when driven with $5.2 \mathrm{kHz}$, a natural frequency of the impacter (Figure 5 (a)). This increase in the system resonance frequency is due to the nonlinear "hardening spring" behavior observed in many dynamic systems involving impacts $[14,13,6,3]$.

As many nonlinear oscillatory systems, the systems involving the nonlinear "hardening springs" are known to exhibit instabilities where a small perturbation of the initial a)
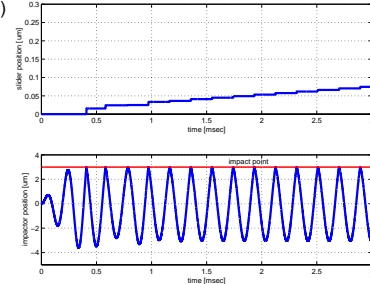
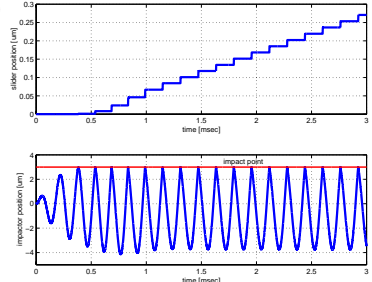

Figure 5. The simulated vibromotor performances.
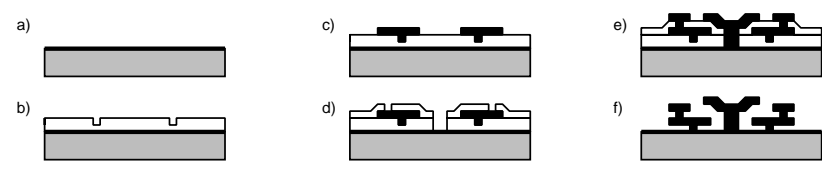

f)

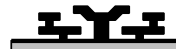

Figure 6. A basic flow of the MUMPS process.

condition $\left(x\left(t_{0}\right), \dot{x}\left(t_{0}\right)\right)$ completely changes the frequency response of the system $[14,13,6,3]$. Our previous investigation [12] discussed that such instabilities could in fact occur in the impacter-slider system as defined in Equations 1 through 8 , since it is likely that the initial position of the impacter mass varies at every operation of the device due to the sticking between the impacter mass and the substrate, and between the impacter tips and the slider side wall. The impactor-slider systems, therefore, must be designed for maximum slider speed, as well as for stable operation at desired external input frequencies [12].

\section{Fabrication and testing}

An array of prototype externally-resonated linear micro vibromotors is fabricated using the three-layer polysilicon surface micro machining process provided by the MCNC MUMPS service, where the bottom polysilicon layer serves as a ground plane, and the middle and the top polysilicon layers are used for micro mechanical structures. Figure 6 illustrates a basic flow of the MUMPS process ${ }^{2}$. A series of figures shows transversal cross sections of the micro linear slider being fabricated. First, the bottom polysilicon layer (referred to as Poly0) is deposited and patterned on a silicon substrate using low pressure chemical vapor deposition (LPCVD), as shown in Figure 6 (a). This is followed by the deposition and patterning of a $0.75 \mu \mathrm{m}$ thick sacrificial layer of LPCVD phosphosilicate glass (PSG). Dimples are wet etched on this PSG layer to reduce friction between the

\footnotetext{
${ }^{2}$ See http: //mems.mcnc. org/mumps. html for details.
} 


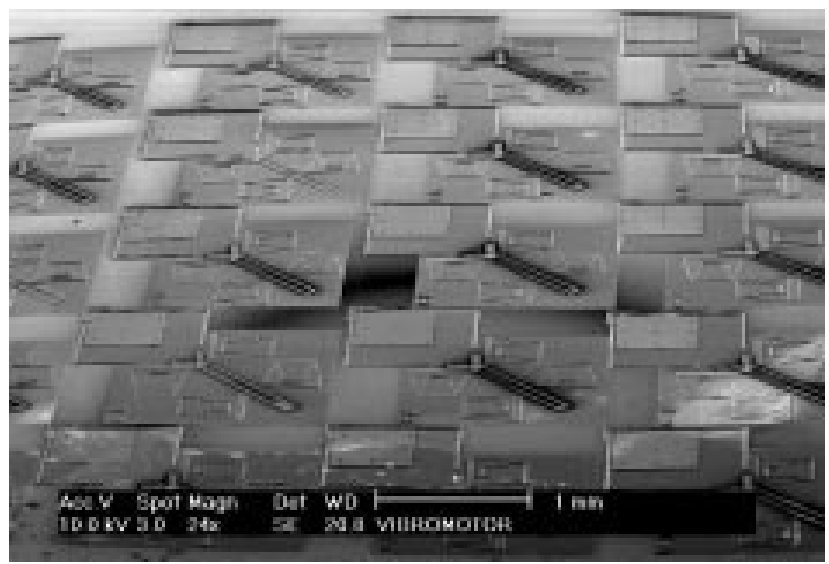

Figure 7. SEM of an array of the externallyresonated micro vibromotors with "dummy" micro components of $500 \mu \mathrm{m} \times 500 \mu \mathrm{m}$.

bottom and middle polysilicon layers at the completion of the fabrication process (Figure 6 (b)). On top of the PSG layer, a $2.0 \mu \mathrm{m}$ thick LPCVD polysilicon layer (referred to as Poly1) is deposited and patterned. Figure 6 (c) shows the cross sectional pattern of the slider made of Poly 1 . After the deposition and patterning of another PSG sacrificial layer (shown in Figure 6 (d)), and a $1.5 \mu \mathrm{m}$ polysilicon layer (referred to as Poly2: shown in Figure 6 (e))), the PSG layers are dissolved in an etching solution (HF), releasing the mechanical structure made of Poly1 and Poly2 (Figure 6 (f)).

Figures 7 and 8 show SEM of an array of the fabricated devices with "dummy" micro components, and a closeup view of one device, respectively. Each vibromotors in the array is designed for a different driving frequency to allow selective activation via an external piezoelectric vibrator. The size of the dummy square components is $500 \mu m \times 500 \mu m$, made with Poly1 layer in the MUMPS process. These dummy micro components are anchored to the substrate with a very thin polysilicon structure which is supposed to be broken with a probe tip at testing. The fabricated devices are tested using the experimental apparatus shown in Figure 9, where the chip containing the device array is attached to two piezo stack vibrators. The horizontal vibrator is to drive the micro linear vibromotors, and the vertical vibrator is to facilitate the gross positioning of micro components on the substrate as described in the next section. This vertical vibrator, however, is not used to obtain the following experimental results.

A sequence of snapshots from a shaking experiment is shown in Figure 10, where the left, middle, and right figure show the initial slider position, after forward motion, and after backward motion, respectively. The control of forward

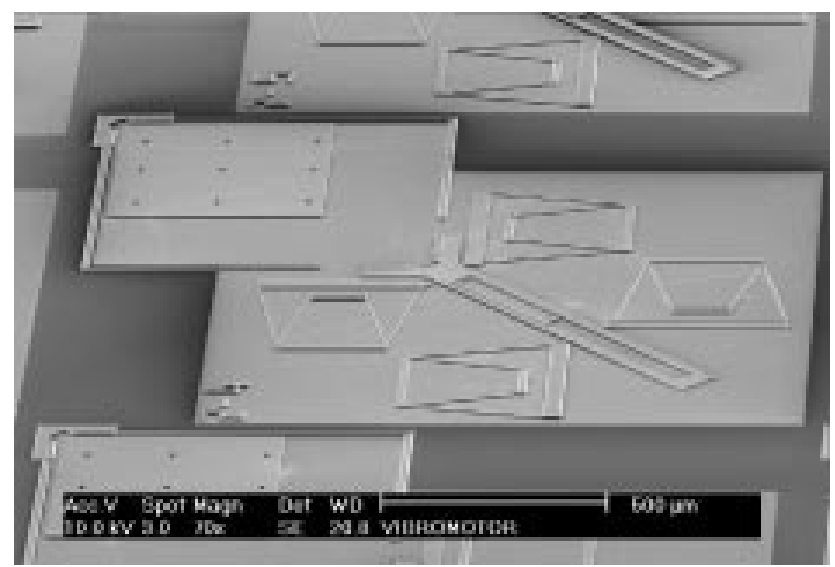

Figure 8. SEM of a close-up view of the externally-resonated micro vibromotor.

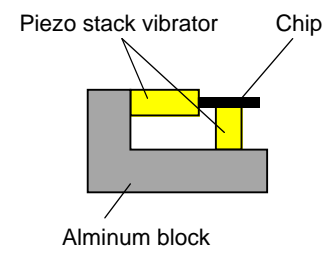

Figure 9. Experimental apparatus for shaking experiments.

and backward slider motion is successfully realized by the selective resonance of forward and backward impacters. It is observed that the full-range slider motion $(300 \mu \mathrm{m})$ occur in approximately a second for most devices in the array, which qualitatively matches the velocity prediction by the correponding simulations. More precise velocity measurement requires high-speed video equipments not available to us at this date. The quantitative validation of simulation results with such equipments is a part of the future work.

\section{Discussion and future work}

This paper proposed a new method for on-substrate fine positioning of micro/meso-scale discrete components, where component positions are finely adjusted using micro linear sliders and fixtures on the substrate. Each micro linear slider is actuated by vibratory impacts exerted by two pairs of micro cantilever impacters. These micro cantilever impacters are selectively resonated by shaking the entire substrate with a piezoelectric vibrator, realizing forward and 


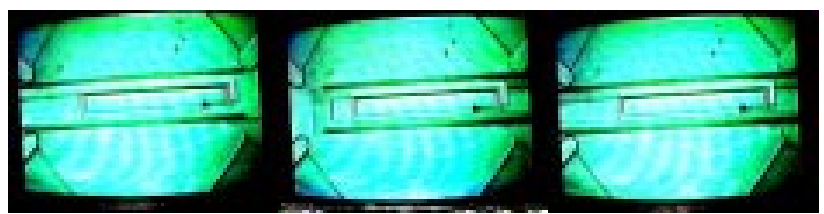

Figure 10. Snapshots from shaking experiments: initial position (left), after forward motion (middle), and after backward motion (right).

backward slider motion to facilitate assembly and disassembly of a micro component on the substrate. An array of the prototype externally-resonated linear micro vibromotors is fabricated using the MCNC MUMPS service. These prototypes are successfully tested for forward and backward motion via external vibration applied by an piezoelectric stack vibrator.

As discussed in Section 1, gross positioning of a micro component needs to be done prior to on-substrate fine positioning using an externally-resonated linear micro vibromotor. Although the gross positioning could be done sequentially in pick-and-place fashion, vibratory palletization [9], a part orienting method common to centimeter-scale mechanical parts, could provide very efficient means of parallel gross positioning of micro components. During the palletization, surface adhesion forces can be virtually eliminated by applying vertical vibration in ultrasonic range as recently reported in [1]. Such vertical vibration can also facilitate the operation of the linear micro vibromotor by reducing the friction between a micro component and the substrate.

The current fine positioning scheme, however, lacks a positive fastening means to secure the attachment of the component to the substrate. Therefore, the design modification of the linear slider, the etched cavity, and/or anchored fixture should be investigated in order to achieve selective fastening and release of a component. For this, the application of removable micro mechanical latching fasteners, or micro "mouse traps," [11] will be considered as a possible fastening means.

\section{Acknowledgments}

The authors acknowledge the partial support for this work provided by National Science Foundation under the grant DMI-9813110.

\section{References}

[1] K. Böhringer, K. Goldberg, M. Cohn, R. Howe, and A. Pisano. Parallel microassembly with electrostatic force fields. In Proceeding of the 1997 IEEE International Conference on Robotics and Automation. IEEE, 1997.

[2] Y.-H. Cho, A. P. Pisano, and R. T. Howe. Viscous damping model for laterally oscillating microstructures. Journal of Microelectromechanical Systems, 3(2):81-87, June 1994.

[3] T. O. Dalrymple. Numerical solutions to vibroimpact via an initial value problem formulation. Journal of Sound and Vibration, 132(1):19-32, 1989.

[4] M. J. Daneman, N. C. Tien, O. Solgaard, A. P. Pisano, K. Y. Lau, and R. S. Muller. Linear microvibromotor for positioning optical components. Journal of Microelectromechanical Systems, 5(3):159-165, September 1996.

[5] R. S. Fearing. Survey of sticking effects for micro parts handling. In Microelectromechanical Systems, pages 212217. IEEE, 1995.

[6] M. S. Heiman, P. J. Sherman, and A. K. Bajaj. On the dynamics and stability of an inclined impact pair. Journal of Sound and Vibration, 114(3):535-547, 1987.

[7] A. S. Laskar and S. Blythe. Epoxy multichip modules: a solution to the problem of packaging and interconnection of sensors and signal-processing chips. Sensors and Actuators A, 36:1-27, 1993.

[8] A. P. Lee and A. P. Pisano. Polysilicon angular microvibromotors. Journal of Microelectromechanical Systems, 1(2):70-76, June 1992.

[9] P. H. Moncevicz, M. J. Jakiela, and K. T. Ulrich. Orientation and insertion of randomly presented parts using vibratory agitation. In A. H. Soni, editor, Proceedings of the ASME 3rd Conference on Flexible Assembly Systems, pages 41-47, New York, NY, September 1991. The American Society of Mechanical Engineers. DE-Vol. 33.

[10] L. Saggere, S. Kota, and S. B. Crary. A new design for suspension systems of linear microactiators. In Proceedings of the 1994 International Mechanical Engineering Congress and Exposition, volume DSC 55-2, pages 671-675. American Society of Mechanical Engineers, 1994.

[11] K. Saitou and M. J. Jakiela. Design of a self-closing compliant "mouse trap" for micro assembly. In 1996 ASME International Mechanical Engineering Congress and Exposition, DSC-Vol. 59, pages 421-426, 1996.

[12] K. Saitou and S. J. Wou. Externally-resonated linear micro vibromotors for micro assembly. In Proceedings of the SPIE Conference on Microrobotics and Micromanipulation, volume 3519, pages 128-139, Boston, MA, November 1998.

[13] S. W. Shaw. Forced vibrations of a beam with one-sided amplitude constraint: theory and experiment. Journal of Sound and Vibration, pages 199-211, 1985.

[14] S. W. Shaw and P. J. Holmes. A periodically forced piecewise linear oscillator. Journal of Sound and Vibration, 90(1):129-155, 1983.

[15] T. Yasuda, I. Shimoyama, and H. Miura. Microrobot actuated by a vibration energy field. Sensors and Actuators A, 43:366-370, 1994. 